\title{
AGES Observations of Abell1367 and its Outskirts
}

\author{
L. Cortese ${ }^{1}$, R. F. Minchin ${ }^{2}$, R. R. Auld ${ }^{1}$, J. I. Davies ${ }^{1}$, B. Catinella ${ }^{2}$, \\ E. Momjian ${ }^{2}$, J. L. Rosenberg ${ }^{3}$, and K. O'Neil ${ }^{4} \&$ The AGES Team \\ ${ }^{1}$ School of Physics and Astronomy, Cardiff University, Cardiff CF24 3AA, UK \\ ${ }^{2}$ Arecibo Observatory, HC3 Box 53995, Arecibo, PR 00612, USA \\ ${ }^{3}$ Harvard-Smithsonian Centre for Astrophysics, 60 Garden Street, Cambridge, MA USA \\ ${ }^{4}$ NRAO, P.O. Box 2, Green Bank, WV 24944
}

\begin{abstract}
The Arecibo Galaxy Environment Survey (AGES, Auld et al. 2006) will map 200 square degrees over the next years using the ALFA feed array at the 305-m Arecibo Telescope. AGES is specifically designed to investigate various galactic environments from local voids to interacting groups and cluster of galaxies. AGES will map 20 square degrees in the Coma-Abell1367 supercluster including the Abell cluster 1367 and its outskirts (up to $\sim 2$ virial radii). In Spring 2006 we nearly completed the observations of 5 square degrees in the range 11:34 $<\mathrm{RA}<11: 54,19: 20<$ Dec $<20: 20$ covering all the cluster core and part of its infalling region reaching a 5 sigma detection limit of $\mathrm{M}(\mathrm{HI}) \sim 4 \times 10^{8} \mathrm{M}_{\odot}$ (assuming a velocity width $\sim 200$ $\left.\mathrm{km} \mathrm{s}^{-1}\right)$ at the distance of Abell1367 ( 92 Mpc). An HI selected sample has been extracted from the datacube obtaining a catalogue of fluxes, recessional velocities, positions and velocity widths. We present a preliminary analysis of the properties of the HI sources and report the discovery of HI diffuse features within interacting groups at the periphery of Abell1367.
\end{abstract}

Keywords. galaxies: evolution-galaxies:clusters: individual: Abell1367-galaxies:interactions

\section{Abell1367}

Abell1367 is a dynamically young local ( $\mathrm{z} \sim 0.023$ ) cluster, currently forming at the intersection of two large scale filaments connecting this system with the Coma and Virgo clusters. It is composed by at least five different substructures that are currently merging each others.

\section{AGES Observations \& Preliminary Results}

In May 2006 we started the observations of the Abell1367 field $(5 \times 4$ degrees, Auld et al. 2006) covering an area of $5 \times 1$ degrees centred on the cluster core. The mean integration time is $\sim 260 \mathrm{sec}$ ( $85 \%$ of the final exposure time) leading to a mean rms of $\sim 1 \mathrm{mJy} / \mathrm{beam}$. A hundreds of HI sources have been detected so far, spanning a wide redshift interval $\left(1000<V<20000 \mathrm{~km} \mathrm{~s}^{-1}\right)$. Diffuse HI features are detected in correspondence with three groups in the outskirts (1-2 degrees from the cluster core) of Abell1367 (CGCG97027, CGCG97041, CGCG97068), suggesting that strong environmental effects are currently perturbing the evolutionary history of these galaxies, well before their infall into the cluster core.

\section{Reference}

Auld, R.R. et al. 2006, MNRAS, 371, 1617. 